\begin{abstract}
A R T I KEL
Poznań

\section{KONTINUITÄT IM WANDEL DER ZEIT. DEUTSCHES KALENDERSCHRIFTTUM UM DIE WENDE ZUM 20. JAHRHUNDERT IN DER PROVINZ POSEN}

\begin{abstract}
Continuity in changing times. The German calendar literature by the turn of the 20th century in the Province of Poznan

Calendars were originally developed for the purpose of temporal orientation and coordination of activities of a group of people. Over time, they have been used as an important means of forming opinions. In regards to that, one cannot underestimate the role of narrative text forms which have been present in calendars since the 16th century and gradually grew in size and importance. The composition of a wide variety of text types and the combination of text and illustration were responsible for the mass distribution of such publications in the 19th century. In the Province of Poznań calendars also enjoyed great popularity. Of special interest is the Evangelical People's Calendar, which was published in Poznan for around 80 years (1861-1941). It accompanied the German population in this area through the major transition points from the end of the 19th to the beginning of the 20th century. In relation to the German calendar literature of the Poznań area, the following questions will be investigated in this article: which turning points of the 20th century were included in the calendar? Which events were considered epochal by the calendar makers? In which form were they presented in the calendars? What was the role of the historical-political and socio-mental context? How was the potential of calendars used to give the readers the sense of continuity and stability by the turn of the century?
\end{abstract}

„Unsere Zeit ist eine Lesezeit, unsere Bildung großenteils eine Lesebildung ${ }^{\text {“1 }}$ mit diesem Satz weist Pastor Hentschel in einem seiner Kalendertexte auf den großen Einfluß der Anfang des 20. Jahrhunderts massenhaft verbreiteten Zeitungen hin. „Dieser merkwürdige papierne Gast [...] hat sich fast in jeder Familie Bürgerrechte erworben“'2 - setzt er im Folgenden fort und voller Wehmut denkt an die „guten

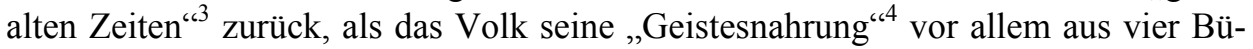

${ }^{1}$ [Vorname unbekannt] Hentschel: Fleißige Bibelleser, in: Evangelischer Volkskalender 1907, S. 37 (im Folgenden abgekürzt EVK zitiert. Im Hinblick auf die getrennte Nummerierung der beiden Kalenderteile werden die Seitenzahlen des Kalendariums in römischen und die des Anhangs in arabischen Zahlen angegeben. In Zitaten wird die Rechtschreibung des Originals beibehalten).

${ }^{2}$ Ebd.

${ }^{3}$ Ebd.

${ }^{4}$ Ebd. 
chern schöpfte: Luthers Katechismus, einem Gesangbuch, einem Erbauungs- oder Predigtbuch und der Heiligen Schrift. Rudolf Schenda zufolge, gehörte im 19. Jahrhundert zum Lesevorrat jeder, nicht nur evangelischen Familie, auch ein Kalender. Aus zwei Teilen zusammengesetzt, dem Kalendarium und einem Anhang mit vielerlei Beiträgen, geschichtlichen und volkskundlichen Schriften sowie Gedichten, Anekdoten und Erzählungen, bildete er lange Zeit die einzige säkulare Literaturquelle des Volkes. ${ }^{5}$

Mit der Übersicht über Feste, Feiertage und Gedenktage sind aber die alten Kalenderhefte weiterhin als zeitliche Landkarten - ,temporal maps' - zu betrachten, die es dem Individuum ermöglichten, sich kurz- und langfristig zurechtzufinden. ${ }^{6}$ In Anbetracht der These von Norbert Elias, dass jede Menschengruppe über ihre eigene Zeit verfügt, die als „Bezugsrahmen [...] dazu dient, innerhalb einer kontinuierlichen Abfolge von Veränderungen von der jeweiligen Bezugsgruppe anerkannte Meilensteine zu errichten" ${ }^{67}$, wird zugleich mit derartigen Druckerzeugnissen ein wesentlicher Teil des Identitätswissens vermittelt und im tagtäglichen Gebrauch eingeübt. Auf das unbestritten große Einübungspotenzial von Kalendern ist es daher zurückzuführen, dass sie sehr oft als wichtige Mittel der Meinungsbildung gebraucht wurden. ${ }^{8}$ In diesem Sinne sollte es nicht verwundern, dass trotz der steigenden Popularität neuer Medien um die Wende des 20. Jahrhunderts weiterhin gerne nach Kalendern gegriffen wurde. Nicht zu übersehen sind zugleich die verschiedenen Bemühungen der Kalendermacher, das lebhafte Interesse an derartigen Druckwerken bei den Lesern zu erhalten.

In einem als ,ein wirklicher Freund “9 bezeichneten Posener Kalender verleugnet Otto Funke - evangelischer Pastor und einer der erfolgreichsten christlichen Volkserzähler des 19. Jahrhunderts - den vermutlich theoretischen oder philosophischen Hintergrund seines Artikels zu den allgemeinen Umständen zu Beginn des 20. Jahrhunderts. Das von dem Autor angestrebte enge Verhältnis zum Publikum mag folgende Textstelle belegen:

\footnotetext{
${ }^{5}$ Rudolf Schenda: Volk ohne Buch. Studien zur Sozialgeschichte der populären Lesestoffe 1770-1910, München 1977, S. 32.

${ }^{6}$ Jürgen P. Rinderspacher: Wochentage im interkulturellen Vergleich, in: Universitas 592/1995, S. 961.

${ }^{7}$ Norbert Elias: Über die Zeit, Frankfurt a.M. 1988, S. 43.

${ }^{8}$ Schon der älteste erhaltene deutschsprachige Kalender in Buchform - der Türkenkalender auf das Jahr 1455 - ist als ein gereimter Kampfaufruf zu betrachten, dessen Inhalt auf zwölf Monate verteilt wurde. In den einzelnen Teilen werden der Papst, der Kaiser und die Fürsten zum Kampf gegen die Türken aufgerufen, die 1453 Konstantinopel erobert hatten und nach Europa vordrangen. Vgl. HansFriedrich Foltin, Britta Schirrmeister: Zeitweiser, Ratgeber, Geschichtenerzähler. Der Funktionswandel des Mediums Kalender in fünf Jahrhunderten, in: Lesekultur. Populäre Lesestoffe von Gutenberg bis zum Internet, hrsg. von Petra Bohnsack, Hans-Friedrich Foltin, Marburg 1999, S. 29f.

${ }^{9}$ Was bringt der neue Diakonissenhaus-Kalender?, in: Blätter aus dem Posener Diakonissenhause 3/1891, S. 5 .
} 
Wir dürfen aber doch wohl unsere Zeit, also den Anfang des 20. Jahrhunderts, einmal näher ins Auge fassen und uns fragen, was das jetzt für eine Zeit ist und wohin der Zeiger an der großen Weltuhr weiset. Es scheint mir sowohl des Kalendermachers, wie der Kalenderleser würdig, darin eine Meinung zu gewinnen. ${ }^{10}$

Ähnliches wird auch mit dem vorliegenden Artikel beabsichtigt. Anhand von ausgewählten Texten aus den deutschsprachigen Kalendern der Provinz Posen aus den Jahren 1899-1918 wird hier der Frage nachgegangen, welche Inhalte über das Druckmedium dem intendierten Publikum in der Umbruchszeit vermittelt werden und welcher Darstellungsformen man sich dabei bedient. Den Ausgangpunkt stellt ein Kalendertext aus der Jahrhundertwende dar, in dem Pastor Münnich aus Kolmar (Chodzież) darüber nachdenkt, wann eigentlich der Schritt zu dem neuen Jahrhundert gemacht wird. Die Frage, ob dies mit dem Neujahrestage 1900 oder 1901 geschehe, bleibt unbeantwortet, in der Skizze kommen jedoch die wichtigsten Tendenzen des Kalenderschrifttums zum Vorschein, die dann im Fortgang des Artikels mit mehreren Beispielen verdeutlicht werden. Eine kurze Darstellung der in dem zu untersuchenden Zeitraum in der Provinz Posen erscheinenden Kalender soll die Einführung zu weiteren Erörterungen bilden.

\section{Deutsches Kalenderschrifttum der Provinz Posen um die Wende zum 20. Jahrhundert im Überblick}

Auf dem Bücher- und Zeitungsmarkt der Provinz Posen gab es Anfang des 20. Jahrhunderts zwei deutschsprachige Kalenderreihen, die über mehrere Jahre hindurch erschienen. Gemeint sind an dieser Stelle der von der Posener Diakonissenanstalt 1874-1941 herausgegebene Evangelische Volkskalender ${ }^{11}$ und der Posener Haus-Kalender für Stadt und Land, der seit 1893 von der Berliner Druckerei des Sonntagsblattes veröffentlicht wurde. Die genauen Erscheinungsjahre des zweiten lassen sich leider nicht feststellen. Während das einzig auffindbare Exemplar des Kalenders aus dem Jahre 1903 stammt, gilt seine dreizehn Jahre ältere Ausgabe in dem Katalog der Staatsbibliothek zu Berlin als Kriegsverlust. Demgegenüber ist der Evangelische Volkskalender fast vollständig erhalten geblieben (Abb. 1). Auf seine Langlebigkeit, die mit der im Laufe der Jahre kontinuierlich steigenden Verbreitung

\footnotetext{
${ }^{10}$ Otto Funcke: Ob die Zeit böse ist?, in: EVK 1909, S. 74f.

${ }^{11}$ Die Entstehung des Kalenders ist auf die Initiative des Divisionspredigers Bork zurückzuführen, der es als notwendig ansah, eine über die Geschichte und die aktuelle Lage der Evangelischen in der Provinz Posen zu berichtende Veröffentlichung zu gründen. Der erste Jahrgang ist 1861 unter dem Titel Evangelischer Kalender für die Provinz Posen erschienen. Angesichts des zurückgehenden Absatzes wurde seine Herausgabe im Jahre 1873 der Posener Diakonissenanstalt überlassen.
} 
einherging ${ }^{12}$, ist auch der besondere Rang des Provinzialkalenders zurückzuführen, den Carl Onnasch folgendermaßen zum Ausdruck bringt:
Ja, wenn des Winters Lampen brennen, Schleichst du dich gern zur Tür hinein, Gibst dich als Hausfreund zu erkennen, Daß jung und alt sich Deiner freun.
$[\ldots]$
Drum weiß mit Pos'ner Land und Leuten Wohl keiner so wie du Bescheid,
Und prächtig weißt du auch zu deuten Der lieben Heimat Lieblichkeit. ${ }^{13}$

Als ein Reservoir an Texten für Jugendliche gilt der Posener Jugendkalender, der 1908 und 1909 in dem Friedrich Ebbeckers Verlag (später Eulitz und Winkler) in Lissa i.P. herausgegeben wurde (Abb. 2). Die von Theodor Krausbauer, dem auch

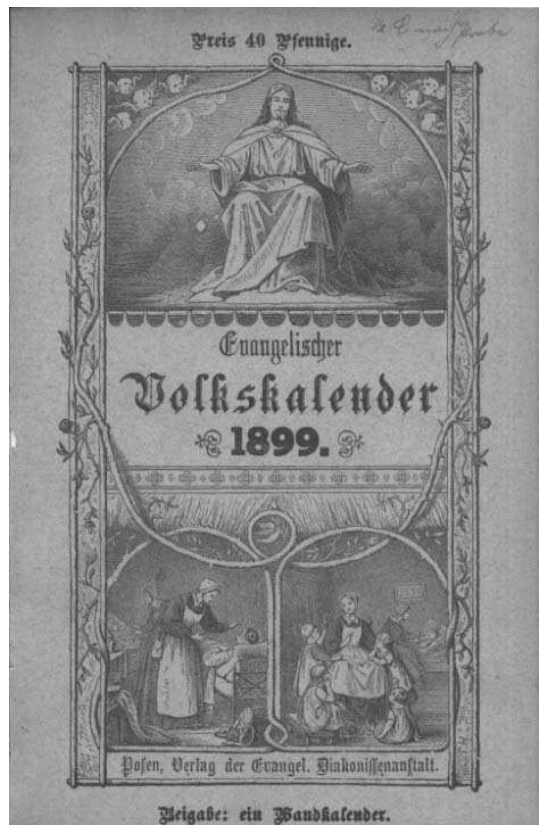

Abb. 1. Titelblatt des Evangelischen Volkskalenders auf das Jahr 1899

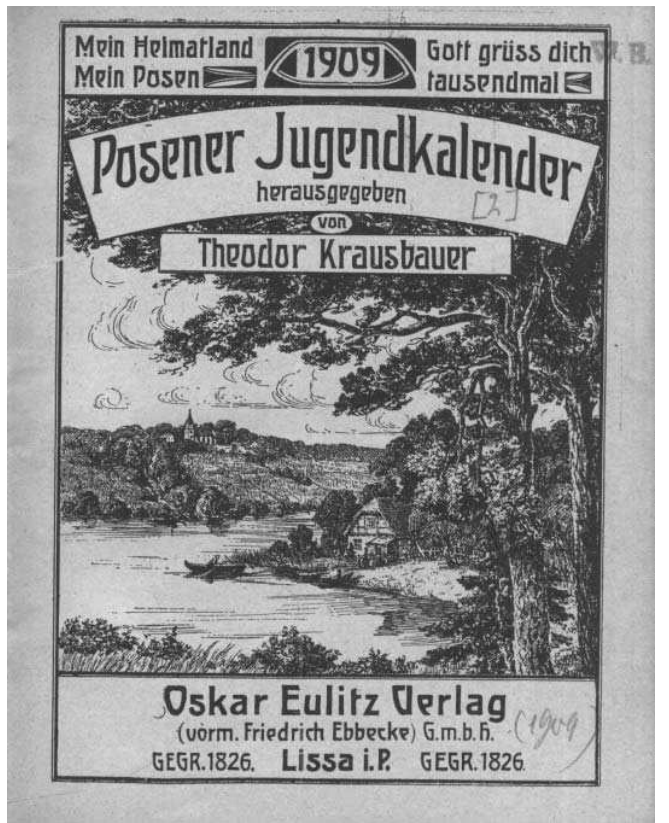

Abb. 2. Titelblatt des Posener Jugendkalenders 1909

\footnotetext{
${ }^{12}$ In dem zu untersuchenden Zeitraum stieg die Auflage des Evangelischen Volkskalenders von 14.500 Exemplaren im Jahre 1899 bis auf 30.000 im Jahre 1918.

${ }^{13}$ Carl Onnasch: Unserem 50jährigen Kalender zum Geleit!, in: EVK 1910, S. II.
} 
unter dem Pseudonym Odo Twiehausen bekannten Schriftsteller und Schulinspektor bearbeitete Publikation kennzeichnet ihr stark ausgeprägter pädagogischer Charakter. Als eine Folgeerscheinung ist der drei Jahre später in demselben Verlag publizierte Posener Jugendland-Kalender zu betrachten, der von zwei Posenern, Hermann Schubert, dem Mittelschullehrer und E. Will redigiert wurde. Die subtile Umformulierung des Kalendertitels lässt sich auch am Inhalt der Veröffentlichung erkennen, die nicht mehr für jugendliche Leser gedacht ist, sondern eher von dem Land ihrer Jugend, dem Posener Land berichtet.

Obwohl es an genauen Angaben zur Reichweite und Auflagenhöhe der oben genannten Publikationen fehlt, kann man annehmen, dass sie nicht nur für die Bewohner der Provinzhauptstadt bestimmt waren, sondern in dem gesamten Gebiet der Provinz Posen in Umlauf gebracht wurden. ${ }^{14}$ Dafür spricht unter anderem die breite inhaltliche Gestaltung der Kalenderhefte. Ganz gewiss gilt die Behauptung für den Evangelischen Volkskalender, der in den im Raum Posen weit gestreuten diakonischen Stationen distribuiert wurde. Die Autoren der gerne als „ein Kind der Heimath" ${ }^{\text {“15 }}$ bezeichneten Veröffentlichung legten großen Wert darauf, dass der Kalender nur für die Gemeinden der Provinz Posen geschrieben wird und „sich über andere Provinzen gar nicht verbreiten [...] lassen [wolle] $]^{\star 16}$. Hier hatte er seine Mission zu erfüllen und ähnlich wie die herausgebende Posener Diakonissenanstalt sollte er nur der Provinz dienen.

Im Hinblick auf das deutsche Kalenderschrifttum der Provinz Posen muss abschließend gesagt werden, dass es - ähnlich wie das preußische Kalenderwesen im Allgemeinen - immer noch unbekannt ist. Volker Bauer zufolge lässt sich der Sachverhalt mit der schmalen Materialbasis erklären. Darüber hinaus besteht die relevante Sekundärliteratur zu diesem Thema aus Aufsätzen, die zum größeren Teil noch vor 1914 herausgegebenen wurden. ${ }^{17}$ Auf den unbestreitbaren Nachholbedarf hat 1988 Edyta Połczyńska in ihrer Monografie ,Im polnischen Wind' hingewiesen, indem sie die bewusstseinsbildende Rolle des deutschen Kalenderschrifttums der Provinz Posen unterstrichen hat. ${ }^{18}$

\footnotetext{
${ }^{14}$ Die Tendenz, Kalender herauszugeben, in denen man sich an Bewohner konkreter Städte oder Kreise wandte, setzte in Großpolen erst nach dem Ersten Weltkrieg ein, als unter anderem folgende Kalenderreihen erschienen: Heimatkalender für den Kreis Fraustadt (1922-1937), Heimatkalender für den Netzekreis (ab 1922) und Heimatkalender des Kreises Kolmar (ab 1941).

${ }^{15}$ Der Evangelische Volkskalender für 1901, in: Blätter aus dem Posener Diakonissenhause 2/1900, S. 4.

${ }^{16}$ Was bringt der neue...? 1891, S. 5.

${ }^{17}$ Vgl. Volker Bauer: Das preußische Kalenderwesen bis zur Mitte des 19. Jahrhunderts, in: Kommunikation und Medien in Preußen vom 16. bis zum 19. Jahrhundert, hrsg. von Bernd Sösemann, Stuttgart 2002, S. 175-192.

${ }^{18}$ Vgl. Edyta Połczyńska: ,Im polnischen Wind'. Beiträge zum deutschen Zeitungswesen, Theaterleben und zur deutschen Literatur im Grossherzogtum Posen 1815-1918, Poznań 1988, S. 151-171.
} 


\section{Ein Kalendertext aus der Jahrhundertwende}

Die um die Wende des 20. Jahrhunderts herrschende Stimmung spiegelt sich deutlich in dem 1899 in dem Evangelischen Volkskalender veröffentlichten Beitrag von Pastor Münnich wider. Der Pastor aus Kolmar teilt hier dem „geneigten Leser“،19 seine Empfindungen mit, die bei ihm das bevorstehende epochale Ereignis auslöst:

Seine Eltern sind einmal mit 1800 in die Welt gekommen und wieder aus der Welt gegangen; mit ihnen wohl noch mancher liebe und treue Gefährte auf irdischer Pilgerfahrt. Es ist ihm beinahe, als hätte er noch einmal Abschied zu nehmen und sei er nun erst recht ein Gast und Fremdling worden auf Erden. ${ }^{20}$

In den nachfolgenden Ausführungen schlägt die Aussage des Textes um, denn wie der Autor an einer Stelle offen gesteht - er bekam von dem Kalendervater den Auftrag, ,zu einem mächtigen Lob- und Dankliede“ ${ }^{\text {‘21 }}$ den Ton anzugeben. In diesem Sinne nimmt Münnich die Jahrhundertwende als eine Gelegenheit wahr, auf das alte Jahrhundert zurück zu blicken, in dem ,der barmherzige Vater [...] unsere liebe Provinz Posen, in der auch dein Haus steht, so wunderbar gesegnet ${ }^{622}$ hat. Nicht ohne Stolz wird hier auf die aktuelle Situation der Evangelischen in der Provinz hingewiesen:

Wie haben doch vor 100 Jahren noch die Väter in unserer lieben Provinz Posen das Wort Gottes, das teuerwerte Evangelium vom Kreuz so mühsam suchen, oft so schmerzlich entbehren müssen! [...] In 100 Jahren sind 115 evangelische Gemeinden neu entstanden und noch viel mehr Kirchen neu erbaut; wird auch, hoff' ich, eh' man 1900 schreibt, noch manche dazukommen. ${ }^{23}$

Das von Münnich mit dem Artikel angestrebte Ziel war aber nicht nur das Loblied ,über eine 100jährige Posener Heils- und Gnadengeschichte“ ${ }^{\text {“24 }}$ erklingen zu lassen, sondern auch seine Leser zu ermahnen: „Und schwere Sünden, geneigter Leser, giebt's doch unter uns; vielleicht sind's auch deine Sünden!‘25 Dem Autor nach, ist das Wohlergehen der Provinz Posen am stärksten durch die zu beobachtende Sucht gefährdet, das Elternhaus früh zu verlassen und in die Ferne zu gehen:

[...] was ist das für ein Ziehen, Laufen, Drängen nach den großen Städten, nach den reichen Gegenden des Westens! Die heiligen Bande der Familienglieder werden locker, die Pietät der Jugend gegen Eltern und Großeltern in der Heimat schwindet, die Ansprüche an das Leben wachsen durch die Genüsse in der Freiheit, die Liebe zu Haus und Herd, zur lieben Heimat nimmt ab. $--^{26}$

\footnotetext{
${ }^{19}$ [Vorname unbekannt] Münnich: Zur Wende des 19. Jahrhunderts, in: EVK 1899, S. 59.

${ }^{20}$ Ebd.

${ }^{21}$ Ebd., S. 60 (Hervorhebung im Original).

${ }^{22}$ Ebd.

${ }^{23}$ Ebd., S. 61.

${ }^{24} \mathrm{Ebd}$.

${ }^{25}$ Ebd. (Hervorhebung im Original).

${ }^{26}$ Ebd., S. 62.
} 
Die Heimattreue wird als eine der christlichen Pflichten betrachtet, denn „,[w]er den irdischen Heimatssinn verliert, der verliert den himmlischen auch leicht. ${ }^{27}$ In diesem Zusammenhang ruft Pastor Münnich seine Leser - die deutschen Einwohner der Provinz Posen - voller Inbrunst auf: „Bleib’ im Lande und nähre dich redlich!“‘28

\section{Von König Friedrich I. zu Bismarck}

Die von Pastor Münnich in dem gerade untersuchten Artikel angewandte Narrationsweise macht die bewährten Tendenzen des Kalenderschrifttums deutlich, die Anfang des 20. Jahrhunderts ungeachtet des Zeitablaufs weiterhin fortgesetzt wurden. Von grundlegender Bedeutung für die Kalendermacher war es nämlich, ein enges Verhältnis zu den Lesern herzustellen. Deshalb werden sie unabhängig von der Textgattung von den Autoren bzw. Erzählern direkt angesprochen und mit weiterführenden Fragen zum Mitdenken eingeladen. Auch die reichlich gebrauchten Sprichwörter sowie Bibel- und Gesangbuchzitate dienen dem Zweck, die Anteilnahme des Publikums aufrecht zu halten. Es soll keine Scheu empfinden und dem Erzähler in seinen Erörterungen hörig folgen.

Eine besonders starke Wirkung versprach man sich von den regionalen Elementen, die sehr oft in einem breiteren Kontext dargestellt wurden. ${ }^{29}$ „Wann sind wir Preußen geworden? Oder wann hat der preußische Adler über unsere jetzige Heimat die schützenden Flügel gebreitet? ‘30 - fragt man in dem 1901 veröffentlichten Kalenderartikel zu dem das neue Jahrhundert eröffnenden 200jährigen Jubiläum des Königreichs Preußen. Im weiteren Verlauf werden die Kalenderleser - die Deutschen der Provinz Posen - dazu aufgefordert, das große Hohenzollernfest gemeinsam zu feiern: „Auf dem Platze soll er sein am 18. Januar als guter Preuße und Deutscher, und soll sein Lied nicht fehlen im Chore derer, die Gott danken für alles, was er Großes an unserm Königshause und unserm Vaterlande gethan hat. ${ }^{\text {‘31 }}$

Die in dem gerade angeführten Beispiel zu beobachtende Verquickung von preußischer und regionaler Geschichte veranschaulicht die Spannung zwischen der regionalen Bindung einerseits und der überregionalen Intention andererseits. Die zwei scheinbar entgegengesetzten, sich ergänzenden Tendenzen - nationale Integration und regionale Heimatidentität - existierten im Zeitalter der deutschen Einigung

\footnotetext{
${ }^{27}$ Ebd.

${ }^{28}$ Ebd.

${ }^{29}$ Der in den Kalendertexten der Provinz Posen zu beobachtende Regionalismus ist als eine literarische Darstellung einer bestimmten Region als geographisch-geschichtlicher Landschaft zu verstehen. Vgl. Norbert Mecklenburg: Erzählte Provinz. Regionalismus und Moderne im Roman, Königstein/ /Taunus 1982, S. 10.

${ }^{30}$ Münnich: Des Königreichs Preußen 200jähriges Jubiläum 18. Januar 1701-1901, in: EVK 1901, S. 31.

${ }^{31}$ Ebd., S. 28 (Hervorhebung im Original).
} 
unter den Hohenzollern parallel und diese Dialektik sei auch für den Regionalismus im 19. Jahrhundert in Deutschland kennzeichnend. ${ }^{32}$

Entsprechend der von Pastor Münnich in seinem Jahrhundertwende-Artikel gemachten Bemerkung: ,Wer von der Vergangenheit nicht lernt, den schlägt die Zukunft $^{\star 33}$ findet man in den deutschen Kalendern der Provinz Posen eine Fülle von Texten, mit denen den Adressaten derartiger Druckerzeugnisse die Geschichte Preußens näher gebracht wird. Es handelt sich hier sowohl um geschichtliche Beiträge, Skizzen und Lebensbilder als auch um Erzählungen und Gedichte. Einen guten Anlass zum Rückblick in die vergangenen Tage boten die im Posener Raum gefeierten Einweihungen der neu erbauten evangelischen Gotteshäuser, die dann in jedem Jahrgang des Evangelischen Volkskalenders aufgelistet wurden. Einen wichtigen Teil dieser Abhandlungen stellten Berichte aus der Geschichte der einzelnen Kirchengemeinden dar, in denen gerne auf die Verfolgung der evangelischen Glaubensanhänger im Posener Land erinnert wird: „Wir wollen's doch nie vergessen, was im 17. und 18. Jahrhundert alles über die evangelische Kirche ergangen ist. Und doch gilt ihr das Wort Psalm 129,2: ,Sie haben mich oft gedränget von meiner Jugend auf, aber sie haben mich nicht übermocht!' 334

In diesem Hinblick erscheint eine Reihe von den in Posener Kalendern verzeichneten Artikeln, mit denen die Leser an konkreten Beispielen belehrt werden, wie sie sich für das Wohlergehen des Vaterlandes - des Deutschen Reiches - und der Evangelischen Kirche einsetzen könnten. Die von Münnich in Erinnerung gerufene Losung: „,Von Gottes Gnade, durch Kampf und Arbeit, zu Gottes Segen'“35 sollte auch den in der Provinz Posen ansässigen Preußen den Weg weisen, dass sie „groß und herrlich bleiben, und immer größer und herrlicher werden unter den Völkern ““36.

An ihre besondere Mission werden die Mitglieder der evangelischen Gemeinden des Posener Landes erinnert, die die Gefahr der Gleichgültigkeit und Trägheit vermeiden sollten und „,der römischen Kirche gegenüber die Augen offen halten müßten ${ }^{\text {، } 37}$. An sie wendet sich im Jahre 1902 der anonyme Autor des Textes Neues evangelisches Leben mit folgenden Fragen:

Sind die Lebens- und Gnadenkräfte der teuren evangelischen Kirche in unsern alten und jungen und jüngsten Gemeinden lebendig? Treten wir mit Glaubensüberzeugung für unsere Kirche ein? Ist es uns eine Herzens- und Gewissenssache, in Haus und Familie, in Beruf und Amt, in Handel und Wandel, Städter und Landmann, Posener von Geburt und Ansiedler aus fernen Landen, alt und jung, mit pro-

\footnotetext{
${ }^{32}$ Vgl. Thomas Serrier: Regionales Selbstbild und nationale Integration. Grundbedingungen und Grenzen der Posener deutschen Identität (1870-1914), in: Nacjonalizm a tożsamość narodowa w Europie Środkowo-Wschodniej w XIX i XX w., hrsg. von Bernard Linek, Kai Struve, Opole u.a. 2000, S. 37.

${ }^{33}$ Münnich: Zur Wende.... 1899, S. 62.

${ }^{34}$ Einiges aus der Leidensgeschichte der evangelischen Kirche in Polen, Böhmen und Ungarn, in: EVK 1910, S. 15.

${ }^{35}$ Münnich: Des Königreichs Preußen.... 1901, S. 30.

${ }^{36}$ Ebd.

${ }^{37}$ Neues evangelisches Leben, in: EVK 1902, S. 50f.
} 
Mit der Einübung in die Heimatliebe und vollkommene Hingabe für das Vaterland setzen die Kalendermacher schon bei den jüngsten Lesern an. Das folgende Gedicht handelt auch von einem Kinderspiel (Abb. 3):
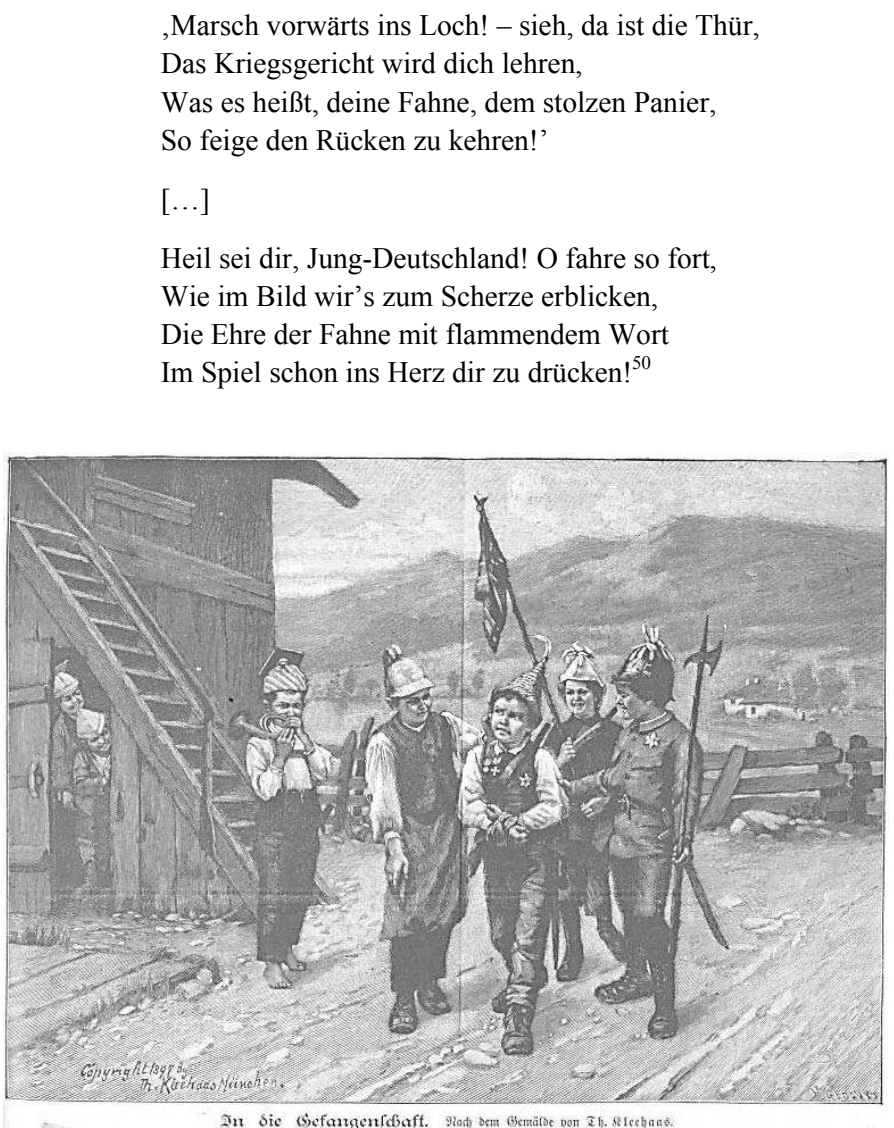

Abb. 3. Illustration zum Gedicht In die Gefangenschaft

Wie es das oben angeführte Beispiel anschaulich macht, war es im Falle von Texten, mit denen man sich an die Jugend wandte, von grundlegender Bedeutung, sie der Aufnahmefähigkeit und der möglichen Aufmerksamkeit des Leserkreises anzupassen. ${ }^{51}$ Deshalb sind die Artikel für Jugendliche meistens kurz und klar aufgebaut. Nicht selten werden dabei die einzelnen Abschnitte zur Erleichterung der Lektüre nummeriert. Auffällig ist die anekdotische Form der Darstellung, auch in

\footnotetext{
${ }^{50}$ M.R. in P.: In die Gefangenschaft, in: EVK 1901, S. 40.

${ }^{51}$ Zur Jugendschriftenfrage!, in: Posener Jugendkalender 1909, S. 93 (im Folgenden abgekürzt PJK zitiert).
} 
Bezug auf geschichtliche Themen. ${ }^{52}$ Es fehlt hier nicht an suggestiven Beschreibungen, die emotionale Teilnahme und dauerhaftes Interesse an dem Stoff erzeugen sollen. So beispielsweise in einem Aufsatz über das Gefecht bei Gostyn, das während des siebenjährigen Krieges zwischen der preußischen Artillerie und der russischen Reiterabteilung stattfand:

Doch vorwärts geht's! An verschiedenen Stellen zugleich dringen die Preußen in die Wagenburg ein. Alles, was ihnen in den Weg kommt, wird voll Erbitterung niedergemacht. [...] Bald jagen die Russen in wilder Flucht davon. Preußische Dragoner verfolgen den fliehenden Feind. Viele werden noch auf der Flucht niedergehauen oder zu Gefangenen gemacht. In einer Stunde war die Blutarbeit getan. ${ }^{53}$

Zur Abwechslung bedient sich Krausbauer der Gedichtform, um über aktuelle Begebenheiten zu sprechen. Von Bismarck - „Wie die Eiche im deutschen Grunde so stark $^{\text {‘54 }}$ - schreibt er wie folgt:

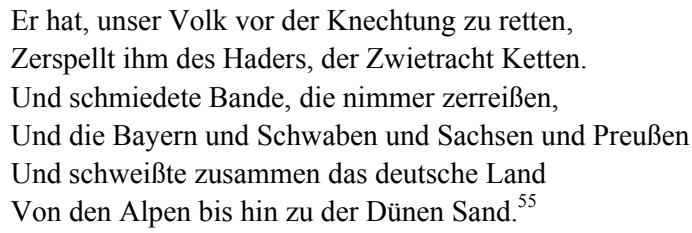

Die Kombination unterschiedlicher Textsorten dient an dieser Stelle der didaktischen Absicht, die Jugend zur Rezeption der zu vermittelnden Inhalte zu motivieren und ihnen zugleich möglichst genaue und intensive Kenntnisse und Denkmuster zu präsentieren. $^{56}$

\section{Der Erste Weltkrieg}

Während Pastor Münnich im Jahre 1899 keine eindeutige Antwort auf die Frage gibt, wann eigentlich von der Jahrhundertwende zu sprechen ist, lässt das den Evan-

\footnotetext{
${ }^{52}$ Vgl. u.a. Napoleon und der Hufschmied von Wengierki, in: PJK 1908, S. 69-72; Der alte Fritz und der Bruchmüller, in: PJK 1909, S. 86-88.

${ }^{53}$ W. Reetz: Das Gefecht bei Gostyn, in: PJK 1908, S. 54f.

${ }^{54}$ Theodor Krausbauer: Mein Bismarck, in: PJK 1909, S. 89.

${ }^{55}$ Ebd.

${ }^{56}$ Die Leser des Posener Jugendkalenders werden schon in dem kalendarischen Teil der Publikation auf die preußische Gedenktage aufmerksam gemacht, die dann parallel mit den familiären Festen zu feiern sind. Auf diese Art werden unter anderem folgende Ereignisse in Erinnerung gerufen: Preußen wird ein Königreich, Kaiser Wilhelm II. geboren, Ende des 7jährigen Krieges, Kaiser Wilhelm I. stirbt, Napoleon I. dankt ab, Der Netze-Distrikt kommt an Preußen, Der 30jährige Krieg beginnt, Friedrich Barbarossa ertrinkt beim Übergang über den Saleph ect. Vgl. Die Monate, in: PJK 1908, S. 4-26.
} 
gelischen Volkskalender des Jahres 1916 eröffnende Gedicht keine Zweifel mehr in dieser Hinsicht:

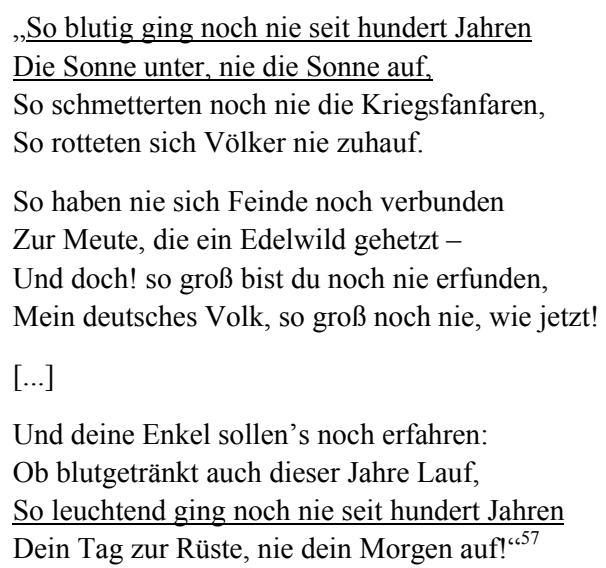

„Krieg, wir haben Krieg!‘58 - schreibt eineinhalb Jahre nach dem Ausbruch des Ersten Weltkriegs der Herausgeber eines Posener Kalenders. „Ein furchtbar ernster Klang, aber auch ein gesegneter Klang, denn er weckte Vaterlandsliebe und heilige Begeisterung, Heldenmut und Opferwilligkeit, stahlharte Kraft und herrliche Einigkeit. ${ }^{\text {“59 }}$ - lesen wir im Folgenden des Aufrufs. Wie erwartet, wird der 1915 auf das Jahr 1916 herausgegebene Evangelische Volkskalender insgesamt dem Kriegsgeschehen gewidmet, das als nationaler Integrationsfaktor interpretiert wird.

Angesichts des gemeinsam zu ertragenden und zu lindernden Leides war es von besonderer Bedeutung den deutschen Einwohnern der ,gering geschätzten Heimatprovinz “60 Posen das Gefühl der Zugehörigkeit zu einem „großen Ganzen“ zu vermitteln. Der Versuch wird unter anderem von Friedrich Just aus Sienno unternommen:

Sie ist uns neu geschenkt, unsere liebe Posener Heimat, seit im November 1914 der Russeneinfall durch unsere Heimatgenossen, den Feldmarschall Hindenburg (geboren in Posen) und seinen Generalstabschef Ludendorff (geboren in Kruschewnia bei Schwersenz), von uns machtvoll abgewehrt ist. Es ist Blut geflossen um unsere Provinz, so wert ist sie geachtet. Und Heimkehr hat die Heimatliebe gehalten in den Herzen. ${ }^{61}$

Die allgemeine Schicksalsverbundenheit veranschaulicht auch die von der Mobilmachung im Posener Land handelnde Geschichte von Pastor Wiese aus Adelnau (Odolanów). Zum Kampf werden hier nämlich die evangelischen Polen aufgerufen:

\footnotetext{
${ }^{57}$ Paul Blau: An der Jahreswende, in: EVK 1916, S. II (Hervorhebung im Original).

${ }^{58}$ Krieg, wir haben Krieg!, in: EVK 1916, S. 113.

${ }^{59}$ Ebd.

${ }^{60}$ Friedrich Just: Von mancherlei Heimkehr, in: EVK 1916, S. 22.

${ }^{61}$ Ebd., S. 24.
} 
„die müssen auch bald mitmachen und kosten, wie französische blaue Bohnen schmecken, sie müssen mit uns bluten, denn schließlich haben wir doch jetzt ein und dasselbe Vaterland zu verteidigen." "62 Über die Familie Rostalski, die auf dem Dorfbahnhof den Hausvater - „,den einzigen Gatten und Vater, Ernährer und Beschützer“63 - verabschiedet, lesen wir wie folgt: „Er zog hinaus ins Feld als ein Held im Granatfeuer und Bajonettkampf, treu seinem Kaiser und Vaterland; sie aber blieben daheim als Helden der Ergebung, des Wartens, der Arbeit und des Gebets. " ${ }^{64}$ So erfährt die Zivilbevölkerung, wie sie sich zu verhalten hat. Auch die Pflichten, die der Krieg den Zurückgebliebenen abverlangt, haben eine integrierende Funktion, denn ,[j] etzt muß jeder Nachbar den allein gelassenen Frauen helfen“65.

Das Kriegsgeschehen wird den Kalenderlesern auf verschiedene Art nahe gebracht. Neben Gedichten, Kalendergeschichten und vielerlei Betrachtungen findet man auch von den Feldgeistlichen verfasste Berichte aus dem Kriegsfelde. Die vielfältige Schilderung vom blutigen aber erfolgreichen Krieg soll bei den Lesern Begeisterung für den Krieg auslösen. Interessanterweise lässt sich erkennen, dass das in dem Posener Kalender dargestellte Bild des Krieges viele Ähnlichkeiten mit den Kriegsberichten von 1870/71 aufweist. Auch in diesem Fall tritt die Absicht in den Vordergrund, den Krieg als ein positives Ereignis zu vermitteln und ein Bewusstsein von gemeinsamer Zugehörigkeit zu erzeugen. ${ }^{66}$

\section{Schlussfolgerungen}

Die deutsche Bevölkerung der Provinz Posen - das intendierte Publikum der oben untersuchten Kalenderreihen - bezeichnet der Historiker Thomas Serrier als eine „kulturell hilfsbedürftige“677 Gemeinschaft. Das für die politisch dominierende Gruppe recht untypische Defizit war unter anderem auf ihre starke Inhomogenität, Zersplitterung und unterschiedlich ausgeprägte Bindung an die Region zurückzuführen. ${ }^{68} \mathrm{Um}$ bei den nach Posen versetzten Verwaltungsbeamten sowie den zur Stützung deutschen Grundeigentums ,importierten“ Siedlern ein Regionalbewusstsein zu schaffen, bediente man sich des aus den, guten alten Zeiten' bekannten Mediums des Kalenders. Über die seit mehreren Generationen in jedem Haushalt vorhandenen und von allen Familienmitgliedern mit Vorliebe gelesenen Druckerzeugnisse machte

\footnotetext{
${ }^{62}$ [Vorname unbekannt] Wiese: Schlichtes Heldentum, in: EVK 1916, S. 3f.

${ }^{63}$ Ebd., S. 7.

${ }^{64} \mathrm{Ebd}$.

${ }^{65}$ Ebd., S. 4.

${ }^{66}$ Gabriele Jaroschka: Lernziel: Untertan. Ideologische Denkmuster in Lesebüchern des Deutschen Kaiserreichs, München 1992, S. 35-68.

${ }^{67}$ Serrier: Regionales Selbstbild.... 2000, S. 41f.

${ }^{68}$ Vgl. Agnieszka Szczepaniak-Kroll: Tożsamość poznańskich rodzin pochodzenia niemieckiego. Losy Bajerleinów i Dittrichów (XVIII-XX w.), Poznań 2010, S. 7.
} 
man die Posener Deutschen mit den dem deutschen Volk gemeinsamen zeitlichen Orientierungspunkten bekannt, deren Bedeutung Norbert Elias hervorhebt. Auf diese Art verfolgte man um die Wende zum 20. Jahrhundert das Ziel, bei den Deutschen der Provinz Posen ein Zusammengehörigkeitsgefühl zu wecken und sie dann mit den Einwohnern anderer preußischer Provinzen zu integrieren. Dank den im Kalendarium verzeichneten Gedenktagen als auch den vielfältigen Darstellungen der nationalen und vaterländischen Geschichte sind nämlich die in dem vorliegenden Artikel besprochenen Kalender als Instrumente der translokalen Integration zu betrachten.

Angesichts der Tatsache, dass die meisten in der Provinz Posen ansässigen Deutschen Protestanten waren, stellte die politisch-nationale Tätigkeit der hiesigen Evangelischen Kirche eine der wichtigsten Voraussetzungen des Integrationsprozesses dar. ${ }^{69}$ Wie Joachim Rogall schreibt, seien die Pastoren der gesamten Provinz wichtige „Repräsentanten und Kristallisationspunkt des Deutschtums ${ }^{\text {“70 }}$. Die dem Amt des Pfarrers inne wohnende Nähe zum Volk prädestinierte ihn auch zur Umsetzung der Politik der Berliner Regierung. In diesem Sinne wirkt auch die literarische Narration des in Posen herausgegebenen Evangelischen Volkskalenders kaum verwunderlich.

Die Konsolidation der Posener Deutschen wurde durch die Versuche gefördert, im Wandel der Zeit mit den über Jahrhunderte hinweg ähnliche Form aufweisenden Kalenderheften bei den Lesern das Gefühl der Kontinuität herzustellen. Besonders deutlich machen dies die den kalendarischen Teil jeden Kalenderjahrgangs eröffnenden Jahreszählungen ${ }^{71}$, die in dem zu untersuchenden Zeitraum im Evangelischen Volkskalender bestehen bleiben. Neben dem Inkarnationsjahr (,seit Christi Geburt") bezieht man sich hier auf solche Ereignisse wie die Einführung des Gregorianischen Kalenders, die Einführung des verbesserten Kalenders, die Erfindung der Buchdruckerkunst, Luthers Reformation, die Erfindung der Dampfmaschinen, die Erhebung des Königreichs Preußen, die französische Revolution, die Geburt Wilhelms II., König von Preußen und den Antritt seiner Regierung und Neuerrichtung des Deutschen Reiches. Demselben Zweck dienen auch die in Kalendern veröffentlichten Behandlungen der nationalen Kriege 1813, 1866 und 1870/71, sowie der dynastischen Geschichte. In jedem Kalenderjahrgang findet man Bilder und Texte, die sich entweder mit Kaisern oder Königen und Feldherren bzw. Reformatoren und evangelischen Geistlichen beschäftigen. Auf diesem Wege erfahren auch die Deutschen der Provinz Posen von der Geschichte der deutschen Minderheit in diesem Gebiet, von ihren zahlreichen Errungenschaften und den Herausforderungen, welche ihnen bevorstehen. Dabei zielt man deutlich auf die Emotionen der Leser ab, denn über die Kalendertexte unterschiedlicher Form und verschiedenen Inhalts sollten sie

\footnotetext{
${ }^{69}$ Olgierd Kiec: Die evangelischen Kirchen in der Wojewodschaft Posen (Poznań) 1918-1939, Wiesbaden 1998, S. 1.

${ }^{70}$ Joachim Rogall: Die Geistlichkeit der Evangelisch-Unierten Kirche in der Provinz Posen 18711914 und ihr Verhältnis zur preußischen Polenpolitik, Marburg/Lahn 1990, S. 240.

${ }^{71}$ Die an dieser Stelle angegebene Zahl der Jahre seit den wichtigen Ereignissen aus der Bibel und Geschichte soll dem Leser auf einen Hieb die Orientierung in der Zeit erleichtern.
} 
in erster Linie lernen, wie sie sich als Vertreter des deutschen Volkes und Angehörige der Evangelischen Kirche zu verhalten haben.

Die Umwandlungen der Zeit in ihrem vollen Umfang macht letztendlich das Layout des Evangelischen Volkskalenders deutlich, als sein 58. Jahrgang zu Beginn des Jahres 1918 in einem völlig neuen Gewand erschien. Das über Jahre beibehaltene Titelbild - die Diakonissenschwester im Dienst der Kranken und Kinder - ersetzte die Abbildung eines von kräftigen Eichenblättern umrahmten Rosenstocks, der seine zarten Zweige hinauf zum Kreuze streckt (Abb. 4). Die Aussage des Bildes wird in dem angehängten Kommentar des Generalsuperintendenten Blau auf folgende Weise erklärt:

Die Eiche ist der deutsche Baum, ihre Knorrigkeit und Kraft redet von kerniger, markiger, deutscher Art. Ein evangelischer Kalender will unser Kalender sein, er grüßt das deutsche Volk mit dem Gruße des Evangeliums. Die Rose unterm Kreuz ist auf deutschem Boden gepflanzt. Hier ist sie am reichsten erblüht. [...] Evangelischer Glaube ist frommes Ergreifen der Kreuzesgnade Christi - ein Rosenstock, der das Kreuz umfaßt - und deutsche Art ist zäh und wetterhart wie Eichenblätter. Und beides zusammen soll dem evangelischen Volkstum Deutschlands auch in harter Zeit und will's Gott, in besseren Zukunftstagen sein Gepräge geben. Sie gehören zusammen, die Eichenblätter und die Rosen und das Kreuz. ${ }^{72}$

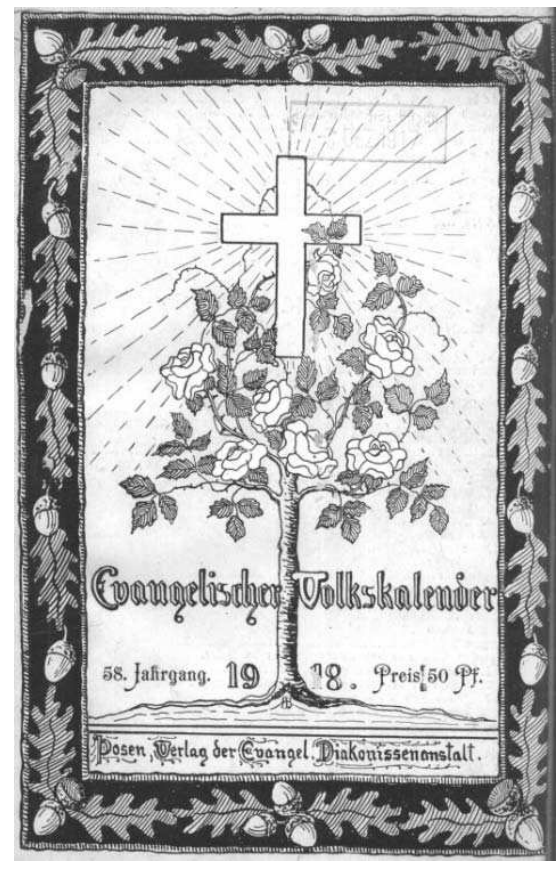

Abb. 4. Das neue Titelblatt des Evangelischen Volkskalenders auf das Jahr 1918

\footnotetext{
${ }^{72}$ Paul Blau: Kreuz und Rose, in: EVK 1918, S. 4.
} 


\section{Literatur}

Der alte Fritz und der Bruchmüller, in: Posener Jugendkalender 1909, S. 86-88 (im Folgenden abgekürzt PJK zitiert).

Bauer, Volker: Das preußische Kalenderwesen bis zur Mitte des 19. Jahrhunderts, in: Kommunikation und Medien in Preußen vom 16. bis zum 19. Jahrhundert, hrsg. von Bernd Sösemann, Stuttgart 2002, S. 175-192.

Blau, Paul: An der Jahreswende, in: Evangelischer Volkskalender 1916, S. II (im Folgenden abgekürzt EVK zitiert. Im Hinblick auf die getrennte Nummerierung der beiden Kalenderteile werden die Seitenzahlen des Kalendariums in römischen und die des Anhangs in arabischen Zahlen angegeben).

Blau, Paul: Kreuz und Rose, in: EVK 1918, S. 3f.

Braut und Bräutigam, in: EVK 1901, S. 43.

Einiges aus der Leidensgeschichte der evangelischen Kirche in Polen, Böhmen und Ungarn, in: EVK 1910, S. 15-18.

Elias, Norbert: Über die Zeit, Frankfurt a.M. 1988.

Der Evangelische Volkskalender für 1901, in: Blätter aus dem Posener Diakonissenhause 2/1900, S. 4.

Foltin, Hans-Friedrich / Britta Schirrmeister: Zeitweiser, Ratgeber, Geschichtenerzähler. Der Funktionswandel des Mediums Kalender in fünf Jahrhunderten, in: Lesekultur. Populäre Lesestoffe von Gutenberg bis zum Internet, hrsg. von Petra Bohnsack, Hans-Friedrich Foltin, Marburg 1999, S. 29-42.

Funcke, Otto: Ob die Zeit böse ist?, in: EVK 1909, S. 73-79.

Hentschel, [Vorname unbekannt]: Fleißige Bibelleser, in: EVK 1907, S. 37-41.

In der Welt ist es dunkel, in: EVK 1901, S. 35f.

Jaroschka, Gabriele: Lernziel: Untertan. Ideologische Denkmuster in Lesebüchern des Deutschen Kaiserreichs, München 1992.

Just, Friedrich: Von mancherlei Heimkehr, in: EVK 1916, S. 19-27.

Kiec, Olgierd: Die evangelischen Kirchen in der Wojewodschaft Posen (Poznań) 1918-1939, Wiesbaden 1998.

Krausbauer, Theodor: Mein Bismarck, in: PJK 1909, S. $89 f$.

Krieg, wir haben Krieg!, in: EVK 1916, S. 113.

Leuchtkäfer in der Ecke, in: EVK 1901, S. 36f.

Mecklenburg, Norbert: Erzählte Provinz. Regionalismus und Moderne im Roman, Königstein/Taunus 1982.

Die Monate, in: PJK 1908, S. 4-26.

M.R. in P.: In die Gefangenschaft, in: EVK 1901, S. 40.

Münnich, [Vorname unbekannt]: Des Königreichs Preußen 200jähriges Jubiläum 18. Januar 1701-1901, in: EVK 1901, S. 28-34.

Münnich, [Vorname unbekannt]: Zur Wende des 19. Jahrhunderts, in: EVK 1899, S. 59-63.

Napoleon und der Hufschmied von Wengierki, in: PJK 1908, S. 69-72.

Neues evangelisches Leben, in: EVK 1902, S. 45-51.

Onnasch, Carl: Unserem 50jährigen Kalender zum Geleit!, in: EVK 1910, S. II.

Połczyńska, Edyta: ,Im polnischen Wind'. Beiträge zum deutschen Zeitungswesen, Theaterleben und zur deutschen Literatur im Grossherzogtum Posen 1815-1918, Poznań 1988.

Reetz, W.: Das Gefecht bei Gostyn, in: PJK 1908, S. 54f.

Rinderspacher, Jürgen P.: Wochentage im interkulturellen Vergleich, in: Universitas 592/1995, S. 960-973.

Rogall, Joachim: Die Geistlichkeit der Evangelisch-Unierten Kirche in der Provinz Posen 1871-1914 und ihr Verhältnis zur preußischen Polenpolitik, Marburg/Lahn 1990.

Schenda, Rudolf: Volk ohne Buch. Studien zur Sozialgeschichte der populären Lesestoffe 1770-1910, München 1977. 
Serrier, Thomas: Regionales Selbstbild und nationale Integration. Grundbedingungen und Grenzen der Posener deutschen Identität (1870-1914), in: Nacjonalizm a tożsamość narodowa w Europie Środkowo-Wschodniej w XIX i XX w., hrsg. von Bernard Linek, Kai Struve, Opole u.a. 2000, S. 37-58.

Szczepaniak-Kroll, Agnieszka: Tożsamość poznańskich rodzin pochodzenia niemieckiego. Losy Bajerleinów i Dittrichów (XVIII-XX w.), Poznań 2010.

Was bringt der neue Diakonissenhaus-Kalender?, in: Blätter aus dem Posener Diakonissenhause 3/1891, S. 5 .

Wiese, [Vorname unbekannt]: Schlichtes Heldentum, in: EVK 1916, S. 3-11.

Zur Jugendschriftenfrage!, in: PJK 1909, S. 93. 